\title{
Porous Materials to Support Bilayer Lipid Membranes for Ion Channel Biosensors
}

\author{
Thai Phung, ${ }^{1}$ Yanli Zhang, ${ }^{1}$ James Dunlop, ${ }^{2}$ and Julie E. Dalziel ${ }^{1}$ \\ ${ }^{1}$ AgResearch, Grasslands Research Centre, Tennent Drive, Private Bag 11008, Palmerston North 4442, New Zealand \\ ${ }^{2}$ MacDiarmid Institute for Advanced Materials and Nanotechnology, Victoria University of Wellington, P.O. Box 600, \\ Wellington 6140, New Zealand
}

Correspondence should be addressed to Julie E. Dalziel, julie.dalziel@agresearch.co.nz

Received 31 March 2011; Accepted 6 July 2011

Academic Editor: Vinod Kumar Gupta

Copyright () 2011 Thai Phung et al. This is an open access article distributed under the Creative Commons Attribution License, which permits unrestricted use, distribution, and reproduction in any medium, provided the original work is properly cited.

To identify materials suitable as membrane supports for ion channel biosensors, six filter materials of varying hydrophobicity, tortuosity, and thickness were examined for their ability to support bilayer lipid membranes as determined by electrical impedance spectroscopy. Bilayers supported by hydrophobic materials (PTFE, polycarbonate, nylon, and silanised silver) had optimal resistance $(14-19 \mathrm{G} \Omega$ ) and capacitance $(0.8-1.6 \mu \mathrm{F})$ values whereas those with low hydrophobicity did not form BLMs (PVDF) or were short-lived (unsilanised silver). The ability of ion channels to function in BLMs was assessed using a method recently reported to improve the efficiency of proteoliposome incorporation into PTFE-supported bilayers. Voltage-gated sodium channel activation by veratridine and inhibition by saxitoxin showed activity for PTFE, nylon, and silanised silver, but not polycarbonate. Bilayers on thicker, more tortuous, and hydrophobic materials produced higher current levels. Bilayers that self-assembled on PTFE filters were the longest lived and produced the most channel activity using this method.

\section{Introduction}

A biological bilayer lipid membrane (BLM) is approximately four nanometres thick consisting of two adjacent monolayer sheets, each composed of molecules with hydrophilic headgroups exposed to water and acyl chains buried into the hydrophobic membrane interior. Integral membrane proteins such as receptors and/or ion channels are responsible for a range of cellular functions that include recognition of pathogens, intracellular signalling, energy transduction, development of energy gradients, and the transport of nutrients and metabolites. Many membrane proteins therefore have potential for use as the sensing entity in biosensors. Two major barriers to the development of biosensors using ion channels are the fragility of biological membranes and their analogues, and the functional requirement of membrane proteins of having solution on both sides of the membrane. Methods that provide enhanced mechanical strength while retaining sensitive electrochemical properties of BLM are being sought to overcome these limitations.

BLM stability can be improved by reducing the area (typically $100-300 \mu \mathrm{m}$ in diameter). Some researchers have addressed this by putting holes in a support structure to create many small BLMs. For example, in films of amorphous Teflon, where it was found that reducing the pore diameter to under $40 \mu \mathrm{m}$ increased BLM stability [1]. Multiple BLMs increase the total BLM area which is important for efficient incorporation of membrane proteins. This has also been achieved by forming a BLM over a polymer matrix in which micro-BLMs are thought to form. Demonstration of BLM formation is determined from either a sufficient membrane capacitance or from functional reconstitution of membrane-spanning peptides or proteins that require the BLM to be $4-5 \mathrm{~nm}$ thick. Polymers of varying porosity and morphology have been found to provide support for BLMs, for example, commercially available filters of polytetrafluoroethylene (PTFE/Teflon, 5 and $10 \mu \mathrm{m}$ pore diameter), polycarbonate $(0.4-8 \mu \mathrm{m}$ pore diameters), polyethylene (PETE), and poly(L-lactic acid) (PLLA) [2-4]. Polycarbonate filters have also been utilised as a support for mixed hybrid BLMs formed over $1 \mu \mathrm{m}$ pore diameters rendered hydrophobic by a gold coating and octadecanethiol [5] in which a glutamate-gated chloride channel was functional [6]. Nylon microfiltration membranes in combination with 
S-layers (crystalline bacterial cell surface layers) produced stable BLMs and functional pore-forming peptide activity [7]. Suspended BLMs supported on highly porous alumina and porous silicon (up to $1 \mu \mathrm{m}$ pore diameter) coated with gold and rendered hydrophobic $[3,8]$ provided a functional environment for OmpF, a bacterial outer membrane protein [9]. BLM formation, determined from capacitance measurements, has also been shown to occur on hydrophobically silanised silicon nitride surface with $200 \mathrm{~nm}$ diameter pores [10].

We recently showed that the pore-forming peptide, gramicidin, and voltage-gated sodium ion channels are functional in BLMs formed on PTFE filters $(5 \mu \mathrm{m}$ pore diameter) [11]. By hydrating the PTFE filter with ionic solution and ion channel proteoliposomes prior to BLM formation, functional reconstitution was enhanced compared with addition of proteoliposomes to the bulk solution. Since this is a convenient means for efficient liposome-BLM fusion, we wanted to know whether other filter materials are suitable BLM supports and if preloading with liposomes enhances incorporation. In this study we examined the physical properties of commercially available membrane filters of polycarbonate, PTFE, nylon, silanised or unsilanised silver, and PVDF, and related this to their ability to support BLMs. The filters were hydrated and BLMs monitored for their stability and longevity by measuring their resistance and capacitance over several hours. The ability of ion channels to function in these BLMs was assessed in filters preloaded with voltage-gated sodium ion channel proteoliposomes. This allowed us to identify which porous materials are able to support BLMs in which ion channels can function, and are therefore of use in biosensor applications.

\section{Materials and Methods}

2.1. Filter Properties. Contact angles were determined from a photograph of a $2 \mu \mathrm{L}$ water droplet placed on a filter [12]. Bubble point (values used were as specified by manufacturers) was used as a measure of the resistance to the flow of fluids through pores of filter materials [13]. The bubble point value is determined by observing when bubbles first begin to emerge on the permeate side or downstream side of a fully wetted membrane filter when pressurized with a gas on the feed (upstream) side of the membrane filter.

2.2. BLM-Support Assembly. Porous supports were constructed by heat-pressing a $3 \mathrm{~mm}$ diameter disc filter over a $1 \mathrm{~mm}$ diameter hole in a polystyrene semi-microcuvette (LP Italiana SPA, cat. no. LPI112117), as detailed in our recent publication using a Teflon filter [11]. The same system was assembled in conjunction with six different filter materials with $5 \mu \mathrm{m}$ average pore diameters: polycarbonate (Osmonics Inc., cat. no. K50SH58050), polytetrafluoroethylene (PTFE, Teflon; Millipore, cat. no. LSWP01300), nylon (Osmonics Inc., cat. no. R50SH04700), polyvinylidene fluoride (Durapore PVDF; Millipore, cat. no. SVLP01300), and silanised and unsilanised metal silver (Sterlitech, cat. no. AG47SP6). Silanised samples of porous silver material were prepared by treating with dimethyldichlorosilane $(\mathrm{BDH})$ at $170^{\circ} \mathrm{C}$ for 2 hours. The filter-cuvette assembly was hydrated with ionic solution and ion channel proteoliposomes by vacuum infiltration at $-75 \mathrm{kPa}$ for 2 hours, as previously described [11].

2.3. Bilayer Formation. The BLM-forming solution contained 5\% (w/w) phosphatidylcholine (PC), extracted from egg yolk [14], and 2\% (w/w) cholesterol (Ajax Finechem, Australia) dissolved in n-octane (Sigma Chemical Co., St Louis, MO, USA), centrifuged at 10,000 rpm for 1 minute and the supernatant collected. $10-20 \mu \mathrm{L}$ was "painted" onto the outer surface of the filter (cis chamber) whereupon a BLM self-assembled.

2.4. Electrical Impedance Spectroscopy. Electrical impedance spectra were obtained using a three-electrode system comprising a platinum counter electrode (cis), a $\mathrm{Ag} / \mathrm{AgCl}$ wire as a reference electrode (cis), and a $\mathrm{Ag} / \mathrm{AgCl}$ measuring electrode (trans). Electrical impedance spectra (EIS) were obtained using PCI14/300 Potentiostat and FAS2 Femtostat manufactured by Gamry Instruments operating under Gamry Framework, Echem Analyst, and EIS300 analysis software (Gamry Instruments, Warminster, PA, USA). Absolute values of the impedance $[\mathrm{Z}](\mathrm{f})$ and the phase angle $\psi(\mathrm{f})$ between voltage and current were recorded within a frequency range of $10^{5}-10^{-2} \mathrm{~Hz}$ optimising for speed with nine data points per decade equally spaced on a logarithmic scale. Data were obtained at zero offset potential applying a $10 \mathrm{mV}$ sinusoidal AC voltage. A nonlinear least square fitting programme was used to fit an equivalent circuit model to the experimental data.

2.5. Ion Channels. Human voltage-gated sodium channel (VGSC) protein was produced using the insect-baculovirus expression system and the preparation enriched for the VGSC, and incorporated into liposomes as previously described [15]. The VGSC proteoliposomes were preloaded into the filter using vacuum infiltration, as detailed previously [11]. Both chambers contained $300 \mathrm{mM} \mathrm{NaCl}, 10 \mathrm{mM}$ HEPES, pH 7.4. Water was purified by a Millipore Milli-Q system (Molsheim, France). Sodium channels were activated using $100 \mu \mathrm{M}$ veratridine (VTD; Sigma) and inhibited using $200 \mathrm{nM}$ tetrodotoxin (TTX; Alomone Labs, Jerusalem Israel). All electrophysiology experiments were carried out at room temperature. Voltage-clamp recordings of ion channel activity were made at a holding potential of $-80 \mathrm{mV}, 0$ and $+80 \mathrm{mV}$ over a period of 15 minutes. A 15-minute control recording was taken prior to addition of the VGSC activator veratridine. Recording and reference $\mathrm{Ag} / \mathrm{AgCl}$ electrodes connected the cell to the amplifier to record ionic currents. VGSC activity was recorded using HEKA EPC7 or EPC9 amplifiers with Pulse v.8.53 and PulseTools v8.8 (HEKA, Lambrecht/Pfalz Germany) and Bruxton Acquire 5.0.1 data acquisition software and analysed using TAC v.4.2.0.

\section{Results and Discussion}

The suitability of each material as a support for a biosensor BLM was determined as the ability to form BLM on 
Polycarbonate

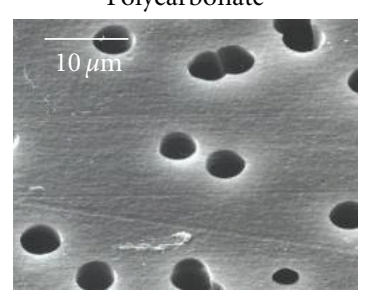

Nylon

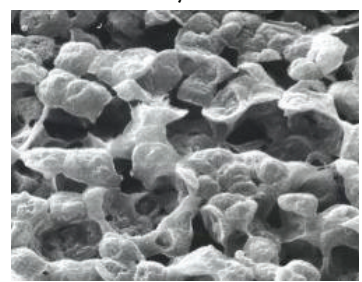

Unsilanised silver

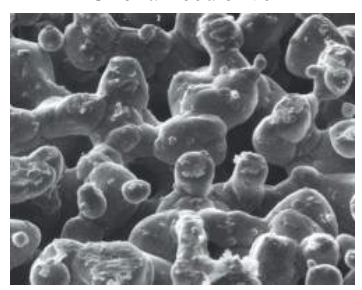

PTFE

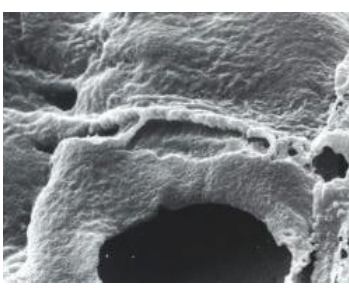

Silanised Ag

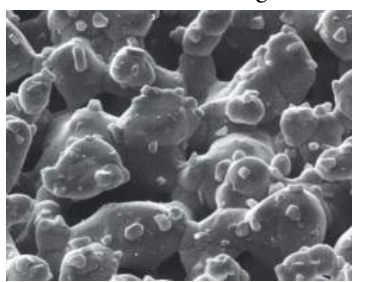

PVDF

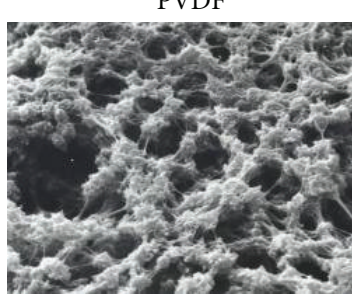

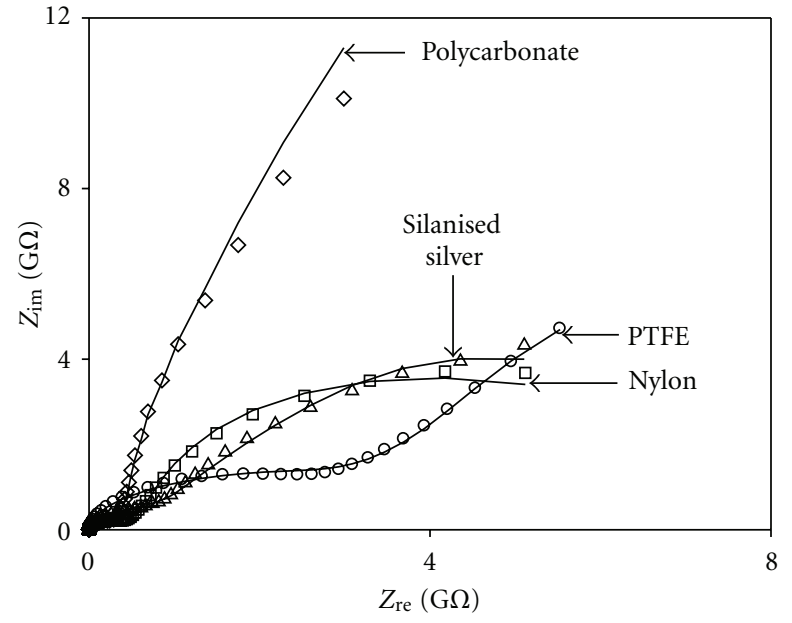

(b)

(a)

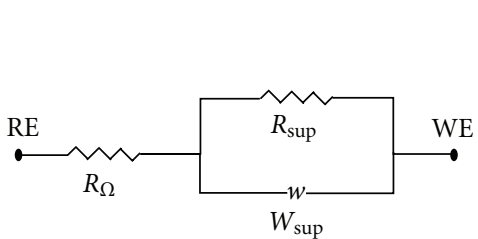

(c)

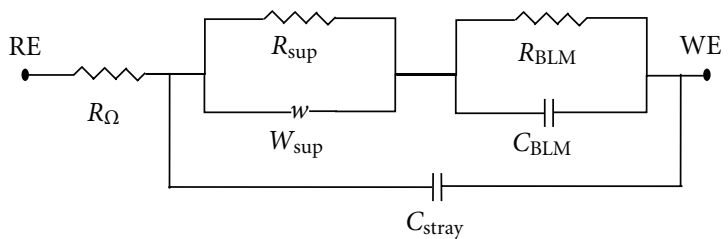

(d)

Figure 1: (a) Electron micrographs showing surfaces and structures of various porous materials (magnification $\times 2000)$. (b) Faradaic impedance spectra are presented in the form of Nyquist plots showing real $\left(Z_{\mathrm{re}}\right)$ versus imaginary $\left(Z_{\text {im }}\right)$ components of BLMs formed on different porous support materials. Electrical circuit models for support (c) and supported BLM (d).

TABLE 1: Properties of filter materials as porous supports.

\begin{tabular}{|c|c|c|c|c|c|c|}
\hline Filter material & Thickness $(\mu \mathrm{m})^{\mathrm{a}}$ & Contact angle (degrees) & Bubble point ${ }^{\mathrm{ab}}$ & BLM formed $\%(\mathrm{n})$ & Longevity ${ }^{\mathrm{c}} \min .(\mathrm{n})$ & $\mathrm{Na}^{+}$current $^{\mathrm{e}} \%(\mathrm{n})$ \\
\hline PTFE & 140 & 141 & 0.70 & $100(15)$ & $101 \pm 7(22)^{\mathrm{d}}$ & $40(10)$ \\
\hline Nylon & $65-125$ & 135 & 0.21 & $71(14)$ & $18 \pm 3(9)$ & $38(8)$ \\
\hline Silanised Ag & 50 & 109 & $\mathrm{n} / \mathrm{a}$ & $92(12)$ & $38 \pm 5(12)$ & $13(8)$ \\
\hline Unsilanised Ag & 50 & 60 & 0.14 & $30(10)$ & $<5(3)$ & - \\
\hline Polycarbonate & 10 & $70-90^{\mathrm{a}}$ & 0.08 & $100(13)$ & $29 \pm 5(14)$ & $0(8)$ \\
\hline PVDF & 125 & $\mathrm{n} / \mathrm{a}$ & $\mathrm{n} / \mathrm{a}$ & $0(10)$ & - & - \\
\hline
\end{tabular}

${ }^{\mathrm{a}}$ Data from manufacturer in methods. ${ }^{\mathrm{b}}$ Alcohol bubble point $\left(\mathrm{kg} . \mathrm{cm}^{-2}\right) .{ }^{\mathrm{c}}$ Mean \pm S.E.M. ${ }^{\mathrm{d}}$ PTFE monitored for up to $2 \mathrm{~h}, 73 \%$ lasted $>2 \mathrm{~h} .{ }^{\mathrm{e}} \mathrm{Number}$ of experiments that gave $\mathrm{Na}^{+}$current increases in supported BLMs preloaded with VGSC liposomes after activation by veratridine and were inhibited by saxitoxin. $\mathrm{n} / \mathrm{a}$, data not available.

their surface and for the preloaded ion channel protein to reconstitute into the BLM and function.

3.1. Properties of Filter Supports. Polycarbonate filters consist of a uniform series of holes on a smooth surface. The pores approximate straight cylindrical holes from one surface to the other. In contrast, apertures of PTFE, nylon, silver, and PVDF filters, are irregular in shape and size (Figure 1(a)). There is no uniform orientation of pores in these filter materials, particularly for nylon and silver, and matrix void spaces vary in their degree of complexity or tortuousity. The thickness of filter materials also varies from $10 \mu \mathrm{m}$ for 

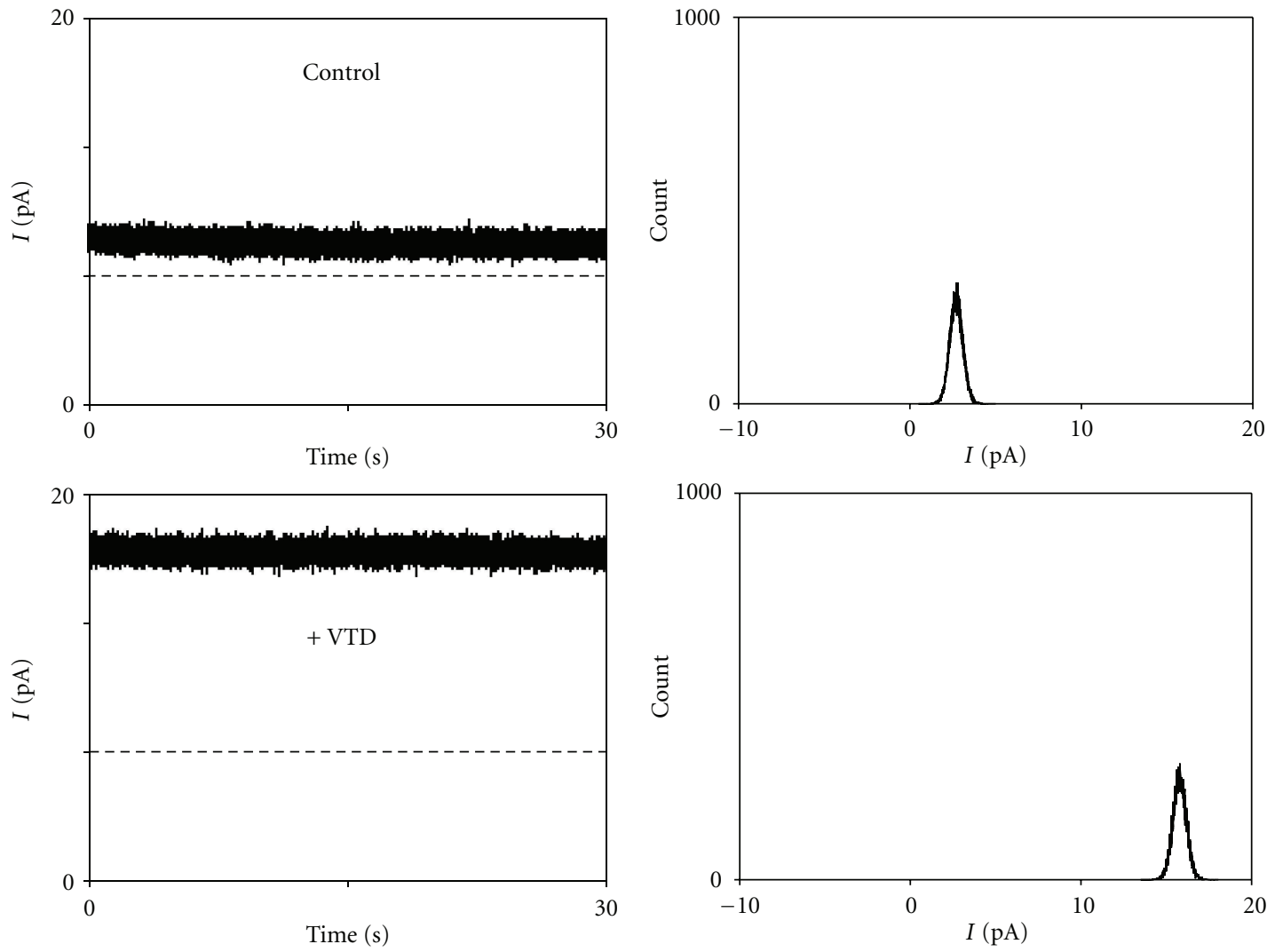

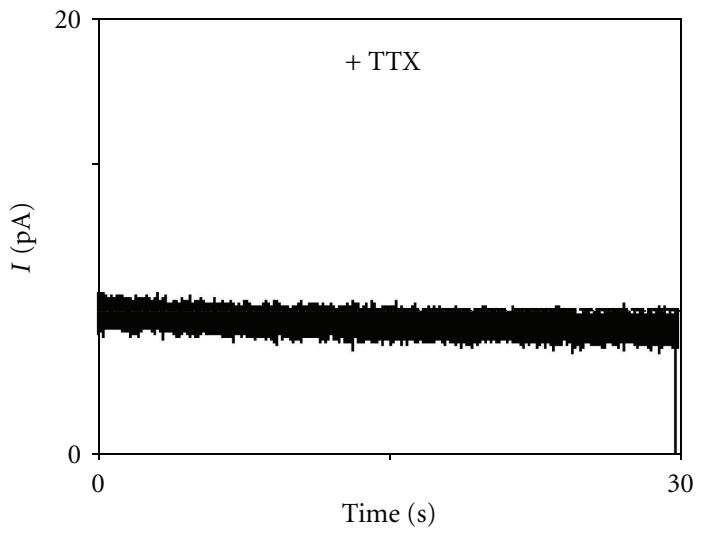

(a)

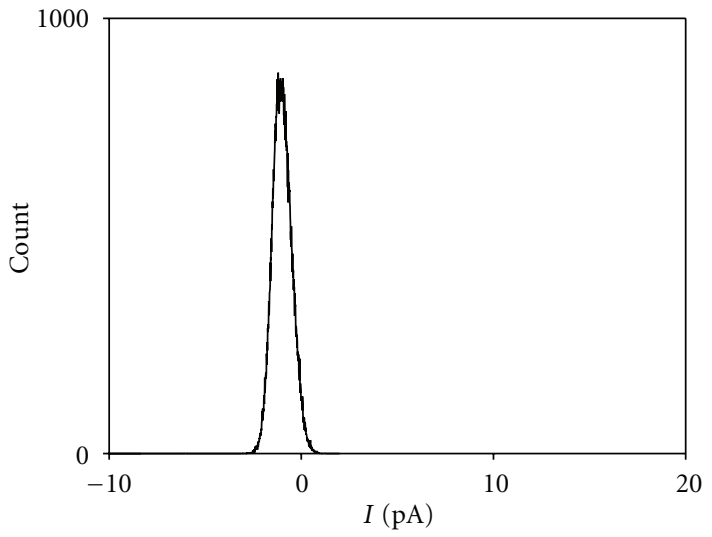

(b)

FIGURE 2: (a) Current recordings from a single experiment are shown for a PTFE filter preloaded with voltage-gated sodium channel (VGSC) liposomes. A BLM was formed on the PTFE filter and the control current level shown after $15 \mathrm{~min}$ at $+80 \mathrm{mV}$. The current level is shown 15 min after addition of $100 \mu \mathrm{M}$ veratridine (VTD) to both sides of the BLM, then $15 \mathrm{~min}$ after addition of $100 \mathrm{nM}$ tetrodotoxin (TTX). (b) Corresponding histograms show the current amplitude distribution over the period of the recording.

TABLE 2: Resistances and capacitances of BLMs on porous supports.

\begin{tabular}{lcccc}
\hline Parameters & PTFE & Nylon & Polycarbonate & Silanised Ag \\
\hline $\mathrm{R}_{\mathrm{BLM}}(\mathrm{G} \Omega)$ & $15.1 \pm 1.6$ & $18.9 \pm 5.8$ & $17.2 \pm 3.3$ & $13.8 \pm 2.3$ \\
$\mathrm{C}_{\mathrm{BLM}}(\mu \mathrm{F})$ & $1.6 \pm 0.1$ & $1.1 \pm 0.2$ & $0.8 \pm 0.1$ & $0.9 \pm 0.1$ \\
$\mathrm{C}_{\text {Stray }}(\mathrm{nF})$ & $37.6 \pm 0.3$ & $38.5 \pm 0.8$ & $36.2 \pm 0.4$ & $36.5 \pm 0.4$ \\
$N$ & 40 & 8 & 12 & 12 \\
\hline
\end{tabular}

Values determined from fit of equivalent model circuit to the data. Data are mean \pm S.E.M.

$\mathrm{R}_{\mathrm{BLM}}$ : membrane resistance, $\mathrm{C}_{\mathrm{BLM}}$ : capacitance of the BLM, $\mathrm{C}_{\text {Stray }}$ : stray capacitance. 
polycarbonate to $140 \mu \mathrm{m}$ for PTFE (Table 1). It is reasonable to assume that for higher degrees of tortuosity, resistance of fluid flow through the filters is also higher. Silanisation did not change the appearance and structure of silver metal filters (Figure 1(a)).

Contact angles determined from a water droplet were used as a relative measure of hydrophobicity [12] (Table 1). Hydrophobic materials would not allow the bath solution to permeate through the filter. It was therefore necessary to apply a negative pressure to remove all air trapped inside the filter before solution would fill the void space. As expected the hydrophobic materials had higher contact angles than hydrophilic materials. PTFE and nylon had the highest contact angles indicating that they are the most hydrophobic materials tested while unsilanised porous silver gave the lowest contact angle and is therefore the least hydrophobic. Silanisation resulted in a higher contact angle for the porous silver indicating an increased degree of hydrophobicity. The contact angle for the polycarbonate and PVDF filters could not be measured because a droplet could not be formed due to water permeation but was obtained from the manufacturer where possible.

Bubble point is a measure of the resistance to the flow of fluids through the filters caused by their thickness and the tortuosity of the void space [13] (Other factors such as uniformity, surface activity, and surface tension can affect bubble point). A high bubble point indicates a high resistance. PTFE and nylon have similar contact angles but the bubble point for the nylon filters was 70\% lower than for PTFE filters (Table 1). Polycarbonate was the thinnest material tested and it had the lowest bubble point. The bubble point of the silanised silver filters was not determined but it would be similar to that of the unsilanised silver material because they have the same pore geometry.

3.2. Bilayer Lipid Membrane Formation. To ascertain whether BLMs were formed on microporous supports, we monitored the electrical properties expected for BLM coverage. Electrical impedance spectroscopy (EIS) provides comprehensive information about the electrical processes of a system under investigation. EIS are generally represented in an equivalent electrical circuit model consisting of parallel and serial elements such as resistance, capacitance, constant phase element, and different types of Warburg elements. The usefulness of EIS to provide quantitative evidence for the presence of BLMs have been demonstrated by many researchers including supported BLMs on PTFE [11], platinum [16], and porous alumina [8]. An electrical circuit model was constructed to fit data obtained from EIS of BLMs on various porous supports (Figures 1(b)-1(d), Table 2). Resistance $\mathrm{R}_{\Omega}$ in serial combination represents the resistance of the solution between the working and reference electrodes. The resistance of support is $R_{\text {sup }}$ while $W_{\text {sup }}$ represents any diffusion of ion across the support. A BLM is in series with the support and comprises a resistor $\mathrm{R}_{\mathrm{BLM}}$ and capacitor $\mathrm{C}_{\mathrm{BLM}}$ in parallel. The equivalent circuit is extended to take into account capacitance $\mathrm{C}_{\mathrm{s}}$ representing the stray capacitance of the measuring cuvette.
A total area of all micro BLMs assumedly formed over void space inside the support structure should be taken into account when calculating capacitances of BLMs. Apart from polycarbonate filter, other porous filters have an ill-defined structure, therefore this measure is unknown. Furthermore, when lipid is applied to a microporous filter, it will ideally form a single BLM, but could also form a series of BLMs in each pore, or a lipid-solvent plug, or a combination of these [4]. Values determined from curve fits for filter supports of PTFE, nylon, polycarbonate, and silanised silver were within ranges for BLM supports reported by other authors for capacitance $[7,17-21]$ and resistance $[8,19,21-24]$.

BLMs self-assembled on most filters except for PVDF and were short lived on silanised silver filters. The data show that the most hydrophobic material (PTFE) produced the most stable BLMs and that they could not be supported by hydrophilic materials such as PVDF and unsilanised silver. Silanisation of silver filter appears to have rendered the surface hydrophobic for lipid interaction (Table 1). The shorter longevity of BLMs in those with lower hydrophobicity, this suggests that strong hydrophobicity of materials is an important factor for the self-assembly of BLMs.

3.3. Voltage-Gated Sodium Channel Activity. To determine whether filter materials besides PTFE could be preloaded with proteoliposomes and ion channels reconstituted into the BLM, their function was assessed in filters that formed robust BLMs, that is nylon, silanised silver, and polycarbonate, and compared with PTFE. Current responses of filters preloaded with voltage-gated sodium ion channel proteoliposomes were monitored before and after addition of the VGSC-activator veratridine, followed by addition of the VGSC-specific inhibitor tetrodotoxin.

Current responses were measured at a holding potential of $-80 \mathrm{mV}, 0$, and $+80 \mathrm{mV}$ over a period of 15 minutes, and was averaged over a one minute recording. The current responses increased from $0 \mathrm{pA}$ (control) to $15-50 \mathrm{pA}$ (after addition of veratridine) over $10-15 \mathrm{~min}$, reversed at $0 \mathrm{mV}$ and were inhibited by tetrodotoxin (Figure 2). The number of filters which responded in this way for a given material were determined (Table 1). The results showed that when VGSC proteoliposomes are preloade into the filter matrices they are able to incorporate into the corresponding BLM in some materials but not others. Nylon and silanised silver filters showed sodium current responses but polycarbonate did not. The 5.6-fold higher BLM longevity for PTFE compared to nylon suggests that PTFE would be the filter material of choice for measuring ion channel activity. The success rate of sodium channel activity in BLMs was lower when formed over thinner and less hydrophobic filter supports (i.e., silanised silver) than on PTFE (Table 1). Materials with low contact angle and low bubble point showed low or no ion channel currents. Thicker supports also produced higher current responses. Polycarbonate was the thinnest filter tested and had the lowest bubble point, suggesting low hydrophobicity which would be consistent with a poor ability to retain proteoliposomes. We did find, however that proteoliposomes added to bath solution 
after BLMs been formed on polycarbonate produced some sodium currents (data not shown), indicating that while they cannot retain preloaded proteoliposomes, these filters can provide a suitable BLM environment for ion channels. This is consistent with the use of polycarbonate filters to support mixed hybrid BLMs that provide a functional environment for glutamate-gated chloride ion channels [6].

\section{Conclusions}

The findings in this study showed that bilayer lipid membranes were most stable when supported by highly hydrophobic materials. Filter supports with greater tortuosity and thickness produced the most ion channel activity when preloaded with proteoliposomes. PTFE was the most versatile filter material of those tested in that it supported long-lived BLMs, and ion channel function when preloaded with ion channel protein. Understanding factors that contribute to BLM stability will help in selection of materials and improved design for customised biosensor systems using membrane proteins.

\section{Acknowledgments}

The authors thank Erwin Berthier for measurements of contact angles. This paper was funded by The New Economy Research Fund, from the Foundation for Research Science and Technology of New Zealand, an AgResearch Postdoctoral Fellowship, and AgResearch Research and Capability and PreSeed Funds.

\section{References}

[1] M. Mayer, J. K. Kriebel, M. T. Tosteson, and G. M. Whitesides, "Microfabricated Teflon membranes for low-noise recordings of ion channels in planar lipid bilayers," Biophysical Journal, vol. 85, no. 4, pp. 2684-2695, 2003.

[2] M. A. Dhoke, P. J. Ladha, F. J. Boerio et al., "Porous membranes for reconstitution of ion channels," Biochimica et Biophysica Acta, vol. 1716, no. 2, pp. 117-125, 2005.

[3] C. Hennesthal, J. Drexler, and C. Steinem, "Membranesuspended nanocompartments based on ordered pores in Alumina," ChemPhysChem, vol. 3, no. 10, pp. 885-889, 2002.

[4] M. Thompson, R. B. Lennox, and R. A. McClelland, "Structure and electrochemical properties of microfiltration filter-lipid membrane systems," Analytical Chemistry, vol. 54, no. 1, pp. 76-81, 1982.

[5] G. Favero, L. Campanella, A. D'Annibale, R. Santucci, and T. Ferri, "Mixed hybrid bilayer lipid membrane incorporating valinomycin: improvements in preparation and functioning," Microchemical Journal, vol. 74, no. 2, pp. 141-148, 2003.

[6] G. Favero, L. Campanella, S. Cavallo et al., "Glutamate receptor incorporated in a mixed hybrid bilayer lipid membrane array, as a sensing element of a biosensor working under flowing conditions," Journal of the American Chemical Society, vol. 127, no. 22, pp. 8103-8111, 2005.

[7] B. Schuster, D. Pum, M. Sára, O. Braha, H. Bayley, and U. B. Sleytr, "S-layer ultrafiltration membranes: a new support for stabilizing functionalized lipid membranes," Langmuir, vol. 17, no. 2, pp. 499-503, 2001.
[8] J. Drexler and C. Steinem, "Pore-suspending lipid bilayers on porous alumina investigated by electrical impedance spectroscopy," Journal of Physical Chemistry B, vol. 107, no. 40, pp. 11245-11254, 2003.

[9] E. K. Schmitt, M. Vrouenraets, and C. Steinem, "Channel activity of OmpF monitored in nano-BLMs," Biophysical Journal, vol. 91, no. 6, pp. 2163-2171, 2006.

[10] X. Han, A. Studer, H. Sehr et al., "Nanopore arrays for stable and functional free-standing lipid bilayers," Advanced Materials, vol. 19, no. 24, pp. 4466-4470, 2007.

[11] T. Phung, Y. Zhang, J. Dunlop, and J. Dalziel, "Bilayer lipid membranes supported on Teflon filters: a functional environment for ion channels," Biosensors and Bioelectronics, vol. 26, pp. 3127-3135, 2011.

[12] R. Förch, H. Schönherr, and A. T. A. Jenkins, Surface Design, Wiley-VCH, Weinheim, Germany, 2009.

[13] F. Hofmann, "Integrity testing of microfiltration membranes," Journal of Parenteral Science and Technology, vol. 38, no. 4, pp. 148-158, 1984.

[14] W. S. Singleton, M. S. Gray, M. L. Brown, and J. L. White, "Chromatographically homogeneous lecithin from egg phospholipids," Journal of the American Oil Chemists' Society, vol. 42, no. 1, pp. 53-56, 1965.

[15] Y. L. Zhang, J. Dunlop, and J. E. Dalziel, "Recombinant human voltage-gated skeletal muscle sodium channels are pharmacologically functional in planar lipid bilayers," Biosensors and Bioelectronics, vol. 22, no. 6, pp. 1006-1012, 2007.

[16] J. Sabo, A. Ottova, G. Laputkova, M. Legin, L. Vojcikova, and H. T. Tien, "A combined AC-DC method for investigating supported bilayer lipid membranes," Thin Solid Films, vol. 306, no. 1, pp. 112-118, 1997.

[17] F. Bordi, C. Cametti, and A. Gliozzi, "Impedance measurements of self-assembled lipid bilayer membranes on the tip of an electrode," Bioelectrochemistry, vol. 57, no. 1, pp. 39-46, 2002.

[18] A. Janshoff, H.-J. Galla, and C. Steinem, in Planar Lipid Bilayers (BLMs) and Their Applications, H. T. Tien and A. OttovaLeitmannova, Eds., Elsevier, Amsterdam, The Netherlands, 2003.

[19] W. Römer, Y. H. Lam, D. Fischer et al., "Channel activity of a viral transmembrane peptide in micro-BLMs: Vpu 1-32 from HIV-1," Journal of the American Chemical Society, vol. 126, no. 49, pp. 16267-16274, 2004.

[20] A. E. Vallejo and C. A. Gervasi, "Impedance analysis of ion transport through gramicidin channels in supported lipid bilayers," Bioelectrochemistry, vol. 57, no. 1, pp. 1-7, 2002.

[21] G. Wiegand, N. Arribas-Layton, H. Hillebrandt, E. Sackmann, and P. Wagner, "Electrical properties of supported lipid bilayer membranes," Journal of Physical Chemistry B, vol. 106, no. 16, pp. 4245-4254, 2002.

[22] S. Gritsch, P. Nollert, F. Jähnig, and E. Sackmann, "Impedance spectroscopy of porin and gramicidin pores reconstituted into supported lipid bilayers on indium-tin-oxide electrodes," Langmuir, vol. 14, no. 11, pp. 3118-3125, 1998.

[23] P. Mueller and D. O. Rudin, "Action potentials induced in biomolecular lipid membranes," Nature, vol. 217, no. 5130, pp. 713-719, 1968.

[24] W. Römer and C. Steinem, "Impedance analysis and singlechannel recordings on nano-black lipid membranes based on porous alumina," Biophysical Journal, vol. 86, no. 2, pp. 955965,2004 


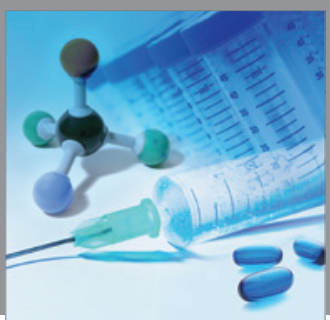

International Journal of

Medicinal Chemistry

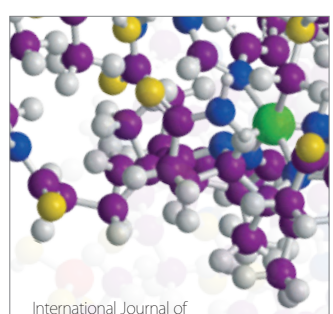

Carbohydrate Chemistry

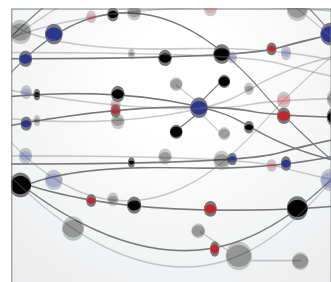

The Scientific World Journal
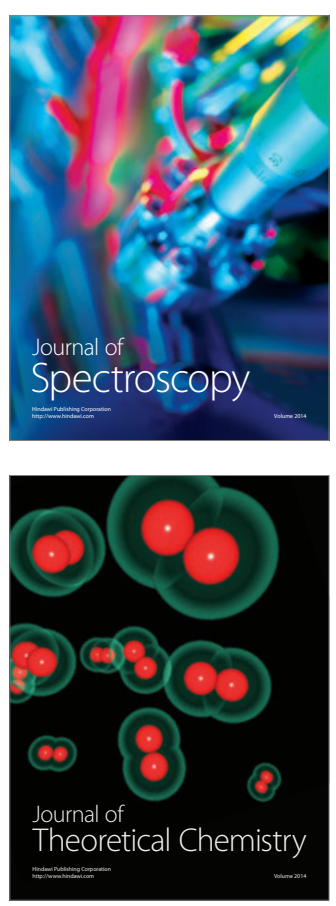
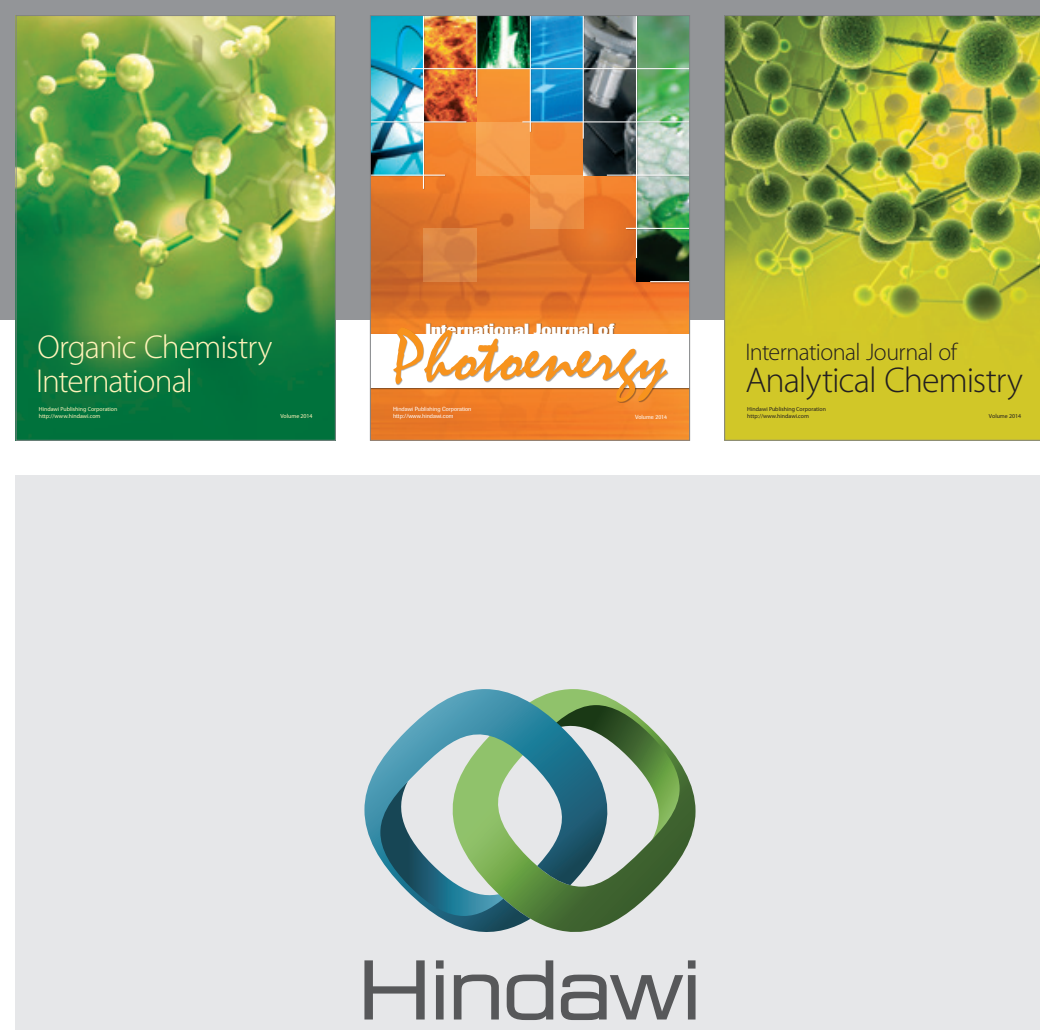

Submit your manuscripts at

http://www.hindawi.com
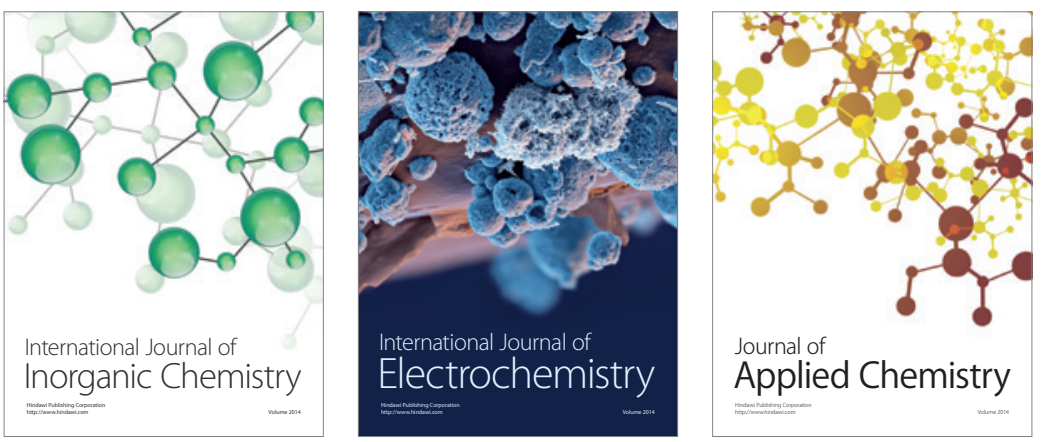

Journal of

Applied Chemistry
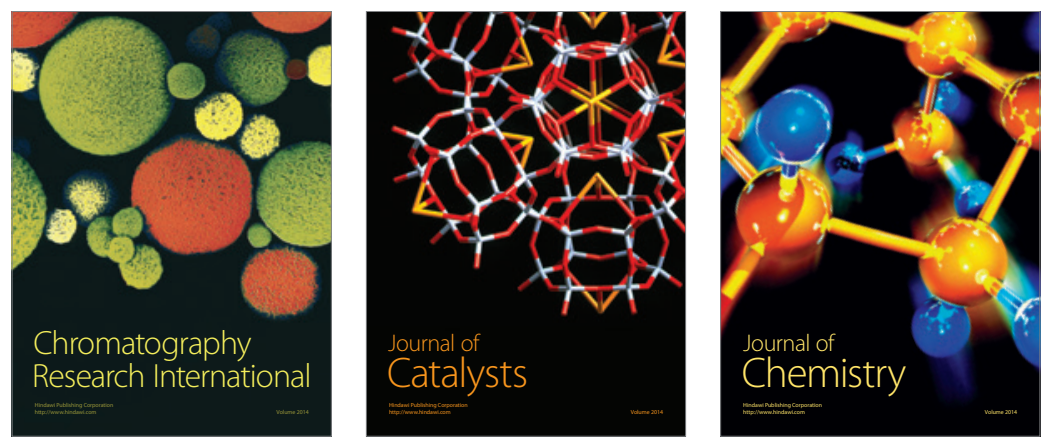
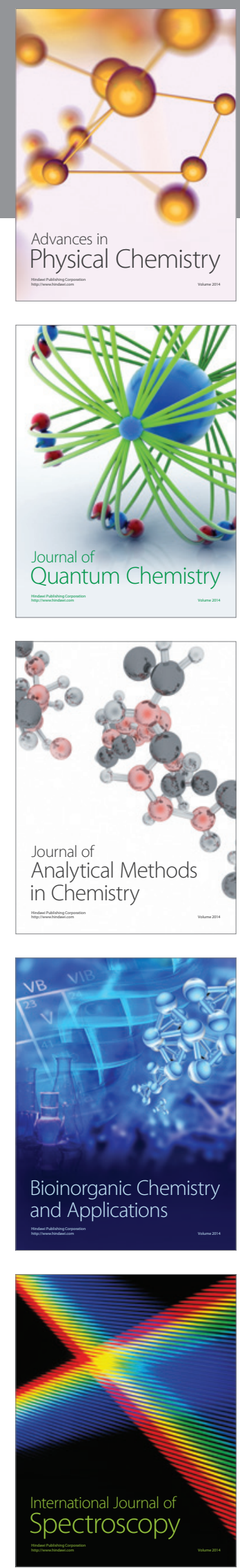